\title{
高性能针刺碳/碳复合材料的制备与性能
}

\author{
刘宇峰 ${ }^{1,2}$, 俸 翔 $^{1}$, 王金明 ${ }^{1}$, 许正辉 ${ }^{1}$, 李同起 ${ }^{1}$, \\ 焦星剑 ${ }^{1}$, 王雅雷 ${ }^{2}$, 熊 翔 $^{2}$
}

(1. 航天材料及工艺研究所先进功能复合材料技术重点实验室, 北京 100076; 2. 中南大学 粉末冶金研究院, 长沙 410083)

摘 要: 为获得高性能针刺碳/碳复合材料, 拓展其应用领域, 通过优化针刺工艺参数, 设计并研制了不同结构参数 的针刺预制体。采用沥青高压致密化工艺将针刺预制体制备成一系列针刺碳/碳复合材料, 研究了针刺碳/碳复合材 料的微观结构、力学性能和热物理性能。结果表明, 针刺预制体的针刺深度、针刺密度以及短/长纤维配比等对碳/ 碳复合材料的力学性能和热物理性能影响显著。当针刺深度为 $12 \mathrm{~mm}$ 、针刺密度为 22 针 $/ \mathrm{cm}^{2}$ 、短/长纤维比例为 $1.0: 4.8$ 时, 针刺碳/碳复合材料表现出优良的综合性能, 拉伸、压缩、弯曲、面内剪切和层间剪切强度分别达到 207 、 228、285、54 和 $28 \mathrm{MPa}$ 。

关 键 词: 碳/碳复合材料; 针刺结构参数; 力学性能; 热物理性能

中图分类号: TB332 文献标识码: A

\section{Preparation and Properties of High-performance Needled C/C Composites}

\author{
LIU Yufeng ${ }^{1,2}$, FENG Xiang ${ }^{1}$, WANG Jinming ${ }^{1}$, XU Zhenghui ${ }^{1}$, LI Tongqi ${ }^{1}$, \\ JIAO Xingjian ${ }^{1}$, WANG Yalei ${ }^{2}$, XIONG Xiang ${ }^{2}$
}

(1. Science and Technology of Advanced Functional Composites Laboratory, Aerospace Research Institute of Materials \& Processing Technology, Beijing 100076, China; 2. Powder Metallurgy Research Institute, Central South University, Changsha 410083, China)

\begin{abstract}
To obtain high performance needled C/C composites, a series of needled non-woven carbon fiber felt with different characteristics was prepared. The needled $\mathrm{C} / \mathrm{C}$ composites were prepared by means of high-pressure impregnation-carbonization, and their microstructure features, mechanical properties and thermo-physical properties of needled $\mathrm{C} / \mathrm{C}$ composites were characterized. The investigation results show that the types of preform structure have obvious effects on the mechanical and thermo-physical properties of the $\mathrm{C} / \mathrm{C}$ composites. When the preform is produced with key characteristics of needling depth at $12 \mathrm{~mm}$, needling density at $22 \mathrm{pin} / \mathrm{cm}^{2}$ and fiber web/weft less ply at 1.0:4.8, the needle $\mathrm{C} / \mathrm{C}$ composites shows excellent comprehensive performance, with tensile strength, compression strength, flexural strength, in-plane shear strength, and interlaminar shear strength of 207, 228, 285, 54 and $28 \mathrm{MPa}$, respectively.
\end{abstract}

Key words: C/C composite; needling characteristic; mechanical property; thermo-physical property

碳/碳复合材料具有高比强度、高比模量、可设

计性强、可加工性好且高温性能优良等特征，在航

收稿日期: 2019-11-29; 收到修改稿日期：2019-12-29

基金项目: 装发共用技术预研项目(41422020106); 科工局基础科研项目(HTKJ2019KL703001); 装发重点实验室基金项目 (61429060102162906002)

Common Technology Project of Equipment Development Department(41422020106); National Defense Basic Scientific Research Project (HTKJ2019KL703001); Key Laboratory Foundation of Equipment Development Department (61429060102162906002)

作者简介: 刘宇峰(1989-), 男, 工程师，博士研究生. E-mail: liuyf123@csu.edu.cn LIU Yufeng(1989-), male, engineer, PhD candidate. E-mail: liuyf123@csu.edu.cn 
空航天领域应用广泛 ${ }^{[1]}$ 。自二十世纪末以来, 世界航 天强国掀起了先进高超声速飞行器研究的热潮, 武 器装备的技战术指标对热防护用碳/碳复合材料在 耐高温、高强、轻质及其低成本化方面提出了更高 要求 ${ }^{[2-5]}$ 。与其它复合材料类似, 碳纤维预制体结构 是决定碳/碳复合材料性能、质量以及生产成本的重 要因素 ${ }^{[6]}$ 。目前常用的碳/碳复合材料预制体结构包 括碳布穿刺、正交三向、三维编织及针刺结构 ${ }^{[7]}$, 其 中碳布穿刺和正交三向无法实现异形件仿形编织; 三维编织纤维利用率低, 因纤维磨损过大而无法编 织复杂形状的预制体。针刺预制体结构是通过网胎 提供的短切纤维在碳布层间的搭接, 不但具有准三 维结构较高的层间性能, 而且具有适合于仿形成 型、连续长纤维方向可设计性强和平面方向纤维利 用率高的优点, 并且制备过程自动化程度高、周期 短、质量稳定, 可高效制备出各种复杂形状的碳纤 维预制体 ${ }^{[8-10]}$ 。目前针刺预制体及其碳/碳复合材料 多应用于火箭发动机喉祄、扩散段、出口锥及飞机 刹车盘等外形简单以及其它承载要求不高的热端部 件 ${ }^{[11-12]}$ 。因传统针刺预制体初始密度低 $(0.20$ $\left.0.45 \mathrm{~g} / \mathrm{cm}^{3}\right)$, 由其致密化制备的碳/碳复合材料力学 性能相较其它类型预制体偏低 ${ }^{[13-15]}$ 。常规的针刺预 制体不能满足高温承载用碳/碳复合材料的使用需 求, 急需开展针刺碳纤维预制体结构参数优化及其 对材料综合性能影响的研究, 以实现高温承载用碳/ 碳复合材料的高性能化和低成本化。

本工作研究了针刺深度、针刺密度以及短/长纤 维配比等针刺织物结构特征参数对针刺预制体及其 碳/碳复合材料的力学性能和热物理性能的影响, 研 制综合性能优良的针刺碳/碳复合材料, 为针刺碳/碳 复合材料在高温承载结构领域的推广应用及热结构 材料的低成本化提供技术支撑。

\section{1 实验方法}

\section{1 材料制备}

采用东丽 $\mathrm{T} 700-12 \mathrm{~K}$ 碳纤维制备的短切纤维网 胎和无纬布交替铺覆 (无纬布 $0^{\circ} / 90^{\circ}$ 循环)制备针刺 预制体。网胎面密度、无纬布面密度、针刺深度 (图 1)以及针刺密度的设计参数见表 1 。其中, 采用 $80 \mathrm{~g} / \mathrm{m}^{2}$ 网胎、 $380 \mathrm{~g} / \mathrm{m}^{2}$ 无纬布、针刺深度 $12 \mathrm{~mm}$ 、 针刺密度 22 针 $/ \mathrm{cm}^{2}$ 工艺参数制备的预制体密度达 到 $0.6 \mathrm{~g} / \mathrm{cm}^{3}$, 比现有工程化应用的针刺预制体密度 显著提高。采用成熟的沥青高压浸渍－碳化致密化工 艺, 针刺预制体及碳/碳复合材料参数如表 1 所示。

\section{2 性能测试}

在航天材料检测与失效分析中心测试针刺预制 体性能和碳/碳复合材料的力学和热物理性能。其中, 参考国军标 GJB1867-94 测试针刺织物性能; 参考 企业标准 DqES415-2005 测试碳/碳复合材料的拉伸 性能, 试样为板状哑铃型, 总长 $130 \mathrm{~mm}$, 试验段尺 寸为 $35 \mathrm{~mm} \times 15 \mathrm{~mm} \times 6 \mathrm{~mm}$; 参考企业标准 DqES293-94

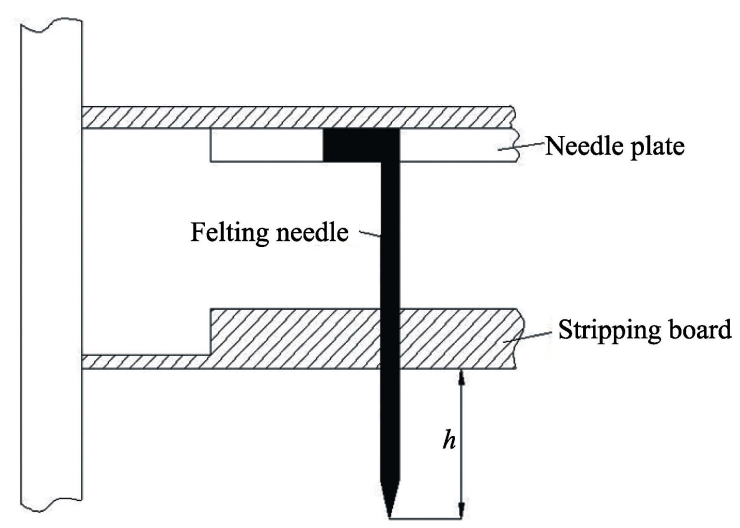

图 1 针刺深度示意图

Fig.1 Schematic diagram of needling depth

表 1 针刺碳/碳复合材料参数

Table 1 Parameters of needled $\mathrm{C} / \mathrm{C}$ composites

\begin{tabular}{cccccccc}
\hline No. & $\begin{array}{c}\rho_{\mathrm{A}} \text {-fiber web/ } \\
\left(\mathrm{g} \cdot \mathrm{m}^{-2}\right)\end{array}$ & $\begin{array}{c}\rho_{\mathrm{A}} \text {-weftless ply/ } \\
\left(\mathrm{g} \cdot \mathrm{m}^{-2}\right)\end{array}$ & $\begin{array}{c}\text { Needling depth/ } \\
\mathrm{mm}\end{array}$ & $\begin{array}{c}\text { Needling density/ } \\
\left(\mathrm{pin} \cdot \mathrm{m}^{-2}\right)\end{array}$ & $\begin{array}{c}\rho_{\text {preform }} / \\
\left(\mathrm{g} \cdot \mathrm{cm}^{-3}\right)\end{array}$ & $\begin{array}{c}\rho_{\mathrm{C} / \mathrm{C}} / \\
\left(\mathrm{g} \cdot \mathrm{cm}^{-3}\right)\end{array}$ & $\begin{array}{c}\text { Porosity/ } \\
\%\end{array}$ \\
\hline A & 200 & 300 & 12 & 22 & 0.31 & 2.073 & 0.677 \\
B-1 & 140 & 340 & 12 & 12 & 0.45 & 2.052 & 0.528 \\
B-2 & 140 & 340 & 12 & 22 & 0.44 & 2.047 & 0.680 \\
B-3 & 140 & 340 & 12 & 27 & 0.44 & 2.052 & 0.681 \\
B-4 & 140 & 340 & 12 & 35 & 0.45 & 2.048 & 0.570 \\
B-5 & 140 & 340 & 15 & 12 & 0.44 & 2.042 & 0.622 \\
B-6 & 140 & 340 & 18 & 12 & 0.44 & 2.040 & 2.361 \\
B-7 & 120 & 300 & 12 & 22 & 0.46 & 2.040 & 1.643 \\
B-8 & 140 & 340 & 12 & 22 & 0.45 & 2.048 & 1.416 \\
C & 80 & 380 & 12 & 22 & 0.6 & 2.016 & 1.127 \\
\hline
\end{tabular}


测试材料的压缩性能, 试样尺寸为 $30 \mathrm{~mm} \times 10 \mathrm{~mm} \times$ $10 \mathrm{~mm}$; 参考国际标准 ASTM C1341-00 测试材料的 弯曲性能, 试样尺寸为 $75 \mathrm{~mm} \times 12 \mathrm{~mm} \times 4 \mathrm{~mm}$; 参考 企业标准 DqES282-97 测试材料的面内剪切性能, 试样轮廓尺寸为 $80 \mathrm{~mm} \times 20 \mathrm{~mm} \times 5 \mathrm{~mm}$, 剪切处试样 宽度为 $12 \mathrm{~mm}$; 参考行业标准 $\mathrm{JC} / \mathrm{T} 773-2010$ 测试层 间剪切性能, 试样尺寸为 $30 \mathrm{~mm} \times 15 \mathrm{~mm} \times 3 \mathrm{~mm}$; 参 考国军标 GJB332A-2004 测试材料的热膨胀系数, 试样尺寸为 $65 \mathrm{~mm} \times 10 \mathrm{~mm} \times 3 \mathrm{~mm}$; 参考国军标 GJB1201.1-91 测试材料的热导率, 试样尺寸为 $\phi 10 \mathrm{~mm} \times 2.6 \mathrm{~mm}$ 。取 5 个以上有效子样测试材料的 力学性能, 测试过程的加载速率为 $1 \mathrm{~mm} / \mathrm{min}$ 。

\section{2 结果与讨论}

\section{1 针刺预制体的性能}

对比分析了不同比例短/长纤维(网胎/无纬布比 例, 其它结构参数相同)的预制体面内/外的拉伸性 能, 如表 2 所示。连续纤维作为承受拉伸载荷的主 体, 随着长纤维占比的增大, 织物的面内拉伸性能 显著提高, 当长纤维比例由 $1.0: 1.5$ 提高至 $1.0: 4.8$ 后, 织物的面内拉伸强度提高了 $88 \%, Z$ 向拉伸强度 略微降低, 为 $11 \%$ 。图 2 为完成致密化的两种短/长 纤维比例碳/碳复合材料的截面形貌, 可以看出预制 体内的无纬布厚度差异明显, $\mathrm{A} / \mathrm{C}$ 试样内的无纬布 层厚度分别约 $0.14 \mathrm{~mm} / 0.26 \mathrm{~mm}$ 。因三种预制体的 针刺密度和针刺深度相同, 即便网胎面密度增大,

表 2 不同纤维比例(网胎/无纬布)针刺织物的性能 Table 2 Mechanical performance of three needled preform

\begin{tabular}{ccccc}
\hline No. & $\begin{array}{c}\rho_{\text {A-fiber web }} / \\
\rho_{\text {A-weftless ply }}\end{array}$ & $\begin{array}{c}\rho_{\text {preform }} / \\
\left(\mathrm{g} \cdot \mathrm{cm}^{-3}\right)\end{array}$ & $\begin{array}{c}\text { XY tensile } \\
\text { strength/MPa }\end{array}$ & $\begin{array}{c}Z \text { tensile } \\
\text { strength/MPa }\end{array}$ \\
\hline A & $1.0: 1.5$ & 0.31 & 17.5 & 0.062 \\
B-8 & $1.0: 2.4$ & 0.44 & 22.0 & 0.060 \\
C & $1.0: 4.8$ & 0.60 & 32.9 & 0.055 \\
\hline
\end{tabular}
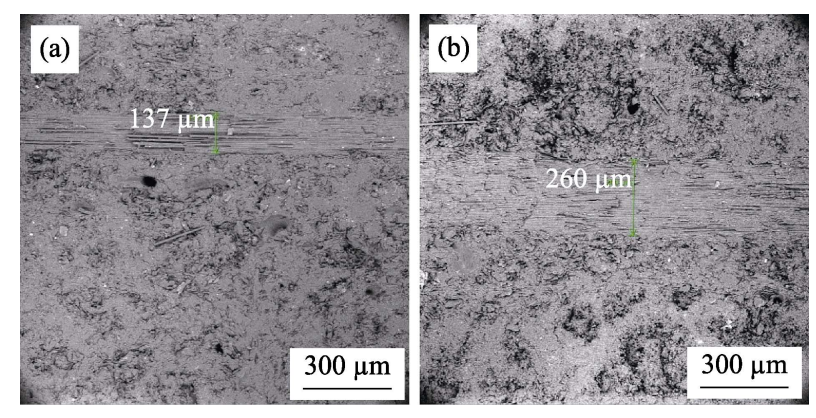

图 2 针刺碳/碳复合材料内的连续布层结构特征 Fig. 2 Microstructure of prepreg in needled C/C (a) Sample A; (b) Sample C
针刺引入 $Z$ 向的短切纤维也不会明显增加, 导致预 制体的 $Z$ 向拉伸强度变化不明显。

\section{2 碳/碳复合材料的性能}

\subsection{1 微观结构}

从图 2 的碳/碳复合材料截面形貌可以看出, 不 同短/长纤维比例预制体内的基体形貌类似，内部孔 隙大部分被沥青碳基体填满, 由沥青在碳化过程中 发生体积变化产生的孔隙和微裂纹主要分布在短切 纤维网胎内, 无纬布层内的孔隙和微裂纹则相对较 少, 这些近似均匀分布的微缺陷有利于材料承载时 重新分配内部应力。在多轮次沥青浸渍一碳化过程中, 逐渐填充了碳/碳复合材料内的孔隙, 直至浸渍通道 被碳基体完全阻塞。在特定工艺条件下, 碳/碳复合 材料的致密化效率及其内部的终态孔隙结构与预制 体结构相关, 从表 1 所示材料终态密度来看, 因初 始密度较低的预制体内部大孔相对较多, 这些大孔 在致密化过程中被沥青碳基体充分填充, 因而完成 致密化后的碳基体含量相对较多, 最终表现为碳/碳 复合材料密度相对较高, 终态开孔率低。其中, 初始 密度最低的 $\mathrm{A}$ 材料最终密度最高, 为 $2.073 \mathrm{~g} / \mathrm{cm}^{3}$; 初始密度最高的 $\mathrm{C}$ 材料最终密度最低, 为 $2.016 \mathrm{~g} / \mathrm{cm}^{3}$ 。

\subsection{2 力学性能}

表 3 为针刺碳/碳复合材料的力学性能。结果表 明针刺织物结构参数对碳/碳复合材料性能影响显 著, 初始预制体密度最高的 $\mathrm{C}$ 材料综合性能最佳。

同种工艺制备的碳/碳复合材料的密度和开孔 率相近, 但因预制体结构参数差异导致材料力学性 能的差异明显。碳/碳复合材料的面内拉伸、面内剪 切和弯曲性能主要由连续纤维的自身强度和沿载荷 方向的连续纤维含量来控制, 压缩性能与碳基体和 纤维的匹配关系相关, 层间剪切性能则主要受 $Z$ 向 纤维的连续性和纤维含量影响。在预制体成型过程 中, 面内连续纤维、网胎以及 $Z$ 向短切纤维的连续 性、完整性以及走向均与针刺成型参数相关, 不同 的针刺结构参数对预制体质量和最终材料性能会有 不同的影响。

1) 针刺深度对材料力学性能的影响 (B-1,B-5, B-6)

针刺深度决定了网胎内的短切纤维在织物厚度 方向上的连续性。针刺深度越深, $Z$ 向短切纤维连续 性越好, 厚度方向引入的 $Z$ 向短切纤维越多。但随 着针刺深度的增大, 针柄进入织物的柄身部分越长, 造成面内方向的连续长纤维偏转, 对无纬布层内的 长纤维连续性和完整性损伤较大。对比针刺深度不 同、其它结构参数相同的三种材料的力学性能可以 发现, 随着针刺深度由 $12 \mathrm{~mm}$ 增加至 $18 \mathrm{~mm}$, 材 
表 3 针刺碳/碳复合材料力学性能

Table 3 Mechanical properties of needled $\mathrm{C} / \mathrm{C}$ composites

\begin{tabular}{|c|c|c|c|c|c|c|c|c|c|}
\hline \multirow[b]{2}{*}{ No. } & \multicolumn{2}{|c|}{ Tensile } & \multicolumn{2}{|c|}{ In-plane compression } & \multicolumn{2}{|c|}{ Flexural } & \multicolumn{2}{|c|}{ In-plane shear } & \multirow{2}{*}{$\begin{array}{c}\text { Interlaminar } \\
\text { shear strength/ } \\
\mathrm{MPa}\end{array}$} \\
\hline & $\begin{array}{l}\text { Strength/ } \\
\mathrm{MPa}\end{array}$ & $\begin{array}{c}\text { Modulus/ } \\
\mathrm{GPa}\end{array}$ & $\begin{array}{l}\text { Strength/ } \\
\mathrm{MPa}\end{array}$ & $\begin{array}{c}\text { Modulus/ } \\
\mathrm{GPa}\end{array}$ & $\begin{array}{l}\text { Strength/ } \\
\mathrm{MPa}\end{array}$ & $\begin{array}{c}\text { Modulus/ } \\
\text { GPa }\end{array}$ & $\begin{array}{l}\text { Strength/ } \\
\mathrm{MPa}\end{array}$ & $\begin{array}{c}\text { Modulus/ } \\
\mathrm{GPa}\end{array}$ & \\
\hline A & 116.0 & 44.85 & 173.1 & 37.76 & 181.5 & 34.56 & 67.9 & 11.08 & 24.6 \\
\hline B-1 & 165.5 & 59.22 & 221.5 & 56.14 & 255.8 & 43.14 & 62.7 & 10.97 & 23.3 \\
\hline B-2 & 151.1 & 57.06 & 201.8 & 51.97 & 249.5 & 40.21 & 58.9 & 10.39 & 24.0 \\
\hline B-3 & 165.0 & 55.34 & 205.2 & 53.02 & 239.1 & 43.36 & 58.9 & 10.68 & 25.0 \\
\hline B-4 & 160.9 & 52.41 & 198.0 & 52.18 & 226.9 & 42.45 & 58.2 & 10.73 & 25.5 \\
\hline B-5 & 150.5 & 56.61 & 198.9 & 49.48 & 211.4 & 39.49 & 59.3 & 10.10 & 24.0 \\
\hline B-6 & 120.4 & 40.99 & 196.4 & 44.72 & 185.1 & 35.36 & 54.7 & 9.93 & 34.6 \\
\hline B-7 & 154.0 & 50.94 & 219.2 & 53.41 & 214.4 & 39.02 & 60.6 & 10.10 & 29.6 \\
\hline B-8 & 154.2 & 51.00 & 200.2 & 54.89 & 221.0 & 40.72 & 60.7 & 10.13 & 26.0 \\
\hline $\mathrm{C}$ & 207.4 & 68.59 & 228.6 & 68.97 & 285.1 & 52.84 & 54.1 & 10.06 & 28.3 \\
\hline
\end{tabular}

料的拉伸、压缩、弯曲和面内剪切强度分别降低了 $27.2 \% 、 11.3 \% 、 27.6 \%$ 以及 $12.7 \%$, 层间剪切强度升 高 $48.4 \%$, 层间剪切强度对针刺深度的变化敏感。

对比典型子样的拉伸载荷曲线(图 3)可以发现, 针刺深度 $12 \mathrm{~mm}$ 试样(B-1)在加载至峰值后出现短 暂的平台期, 而后再呈一定斜率下降, 表现出“伪塑 性断裂特征”, 其断口形貌存在大量的纤维拔出现 象(图 4(a)), 发挥了纤维的增韧效果。而针刺深度

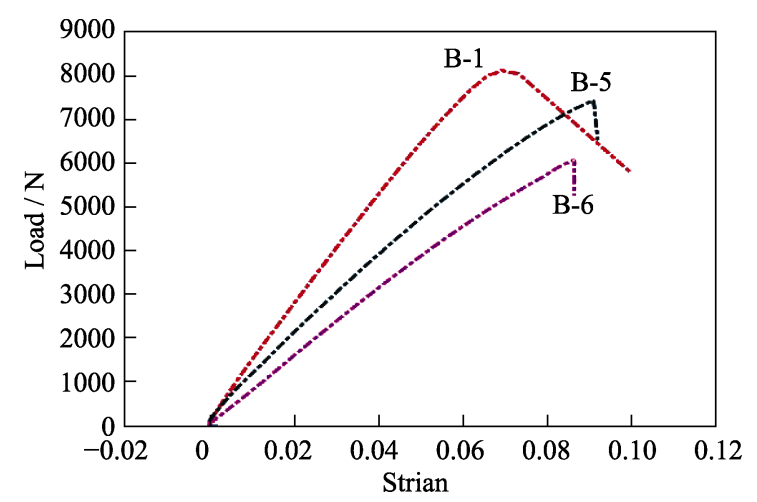

图 3 典型碳/碳复合材料的拉伸载荷-应变图

Fig. 3 Tensile load-strain behavior of needle $\mathrm{C} / \mathrm{C}$ samples
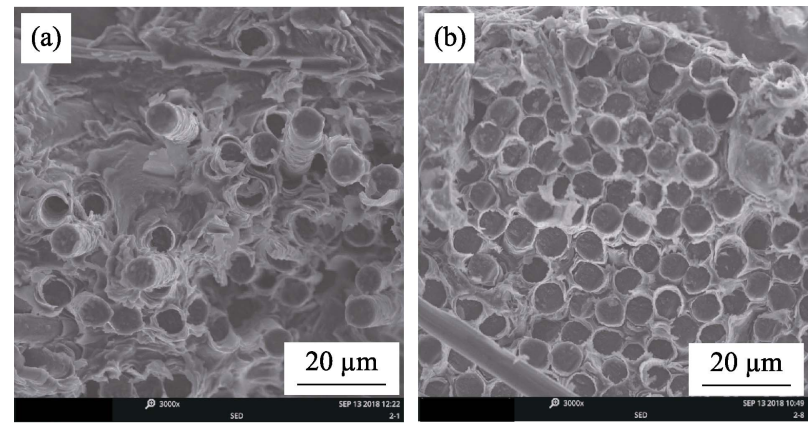

图 4 不同针刺深度碳/碳复合材料断口形貌对比

Fig. 4 Fracture surface micro-morphologies of needle $\mathrm{C} / \mathrm{C}$ composites

(a) B-1; (b) B-6
$15 \mathrm{~mm}(\mathrm{~B}-5)$ 和 $18 \mathrm{~mm}(\mathrm{~B}-6)$ 的试样在加载至峰值后 瞬时卸载, 其拉伸断口平整(图 4(b)), 呈脆性断裂特 征。针刺深度浅的 B-1 材料内的连续纤维受针柄的 影响小, 在承载方向上更为平直, 受载过程各纤维 协调承载, 表现出较好的韧性, 弹性模量较高; 在 针刺深度较深的 B-5 和 B-6 样品中, 连续纤维受针 柄进入的影响, 发生偏转, 表现出碳基体的脆性。

2)针刺密度对材料力学性能的影响 (B-1, B-3, B-4)

针刺密度指单位面积内的针刺次数。针刺密度 越高, 单位面积内引入 $Z$ 向的短切纤维越多, 层间 连续性越好。但随着针刺密度的增大, 刺针对碳布 面内连续纤维造成的损伤越多, 面内性能降低。对 比针刺密度不同、其它结构参数相同的三种材料的 力学性能发现, 随着针刺密度由 12 针 $/ \mathrm{cm}^{2}$ 增加至 35 针 $/ \mathrm{cm}^{2}$, 材料的拉伸、压缩、弯曲和面内剪切强 度分别降低了 $2.77 \% 、 10.6 \% 、 11.3 \%$ 和 $7.18 \%$, 层间 剪切强度升高 $9.44 \%$ 。针刺密度和针刺深度的变化 对材料力学性能的影响趋势一致, 但影响程度存在 差异, 针刺深度变化对碳/碳复合材料性能的影响显 著大于针刺密度变化的影响, 该试验结果与文献 [16]中针刺碳/碳复合材料性能的响应曲面法显著性 的分析结果一致。

3)短/长纤维比例对材料力学性能的影响 (A, B-8, C)

针刺预制体由网胎和无纬布按一定规律交替铺 层后, 通过刺针将网胎内的短切纤维引入 $Z$ 向, 由 无纬布内的连续纤维实现面内高承载, 由网胎内的 短切纤维实现层间连续。面密度是网胎和无纬布的 关键特征参数, 面密度越高, 单层内纤维就越多, 布层越厚。对比表 2 所述三种短/长纤维比例不同、 
其它结构参数相同的预制体对应的碳/碳复合材料 的性能发现, 随着短/长纤维比例由 $1.0: 1.5$ 变化至 $1.0: 4.8$, 材料的拉伸、压缩以及弯曲强度分别提高 了 $78.8 \%$ 、32.0\%以及 $57.0 \%$, 层间剪切强度提高 $15.0 \%$, 但面内剪切强度降低 $9.2 \%$ 。对比三种材料 的典型拉伸试样断口形貌(图 5), A 板材试样断口处 的平断形貌较多; B-8 和 C 板材试样断口的纤维拔 出现象较为明显, 发挥了纤维的韧性, 材料强度和 模量比 A 样品明显提高。

综上, 随着短/长纤维比例的变化, 若连续布层 在预制体内的含量越高，则材料的拉伸、压缩和弯 曲性能明显提高; 因 $\mathrm{C}$ 板材网胎面密度低, 针刺密 度和深度相同的情况下, 引入的 $Z$ 向短纤维相对较 少，层间剪切强度没有出现类似拉/压/弯性能明显 提高的现象; 因 $\mathrm{C}$ 板材网胎面密度低, 材料面内的 无序短切纤维较少, 受面内剪切载荷时, 剪切面上 的短纤维也相对较少, 面剪强度低。
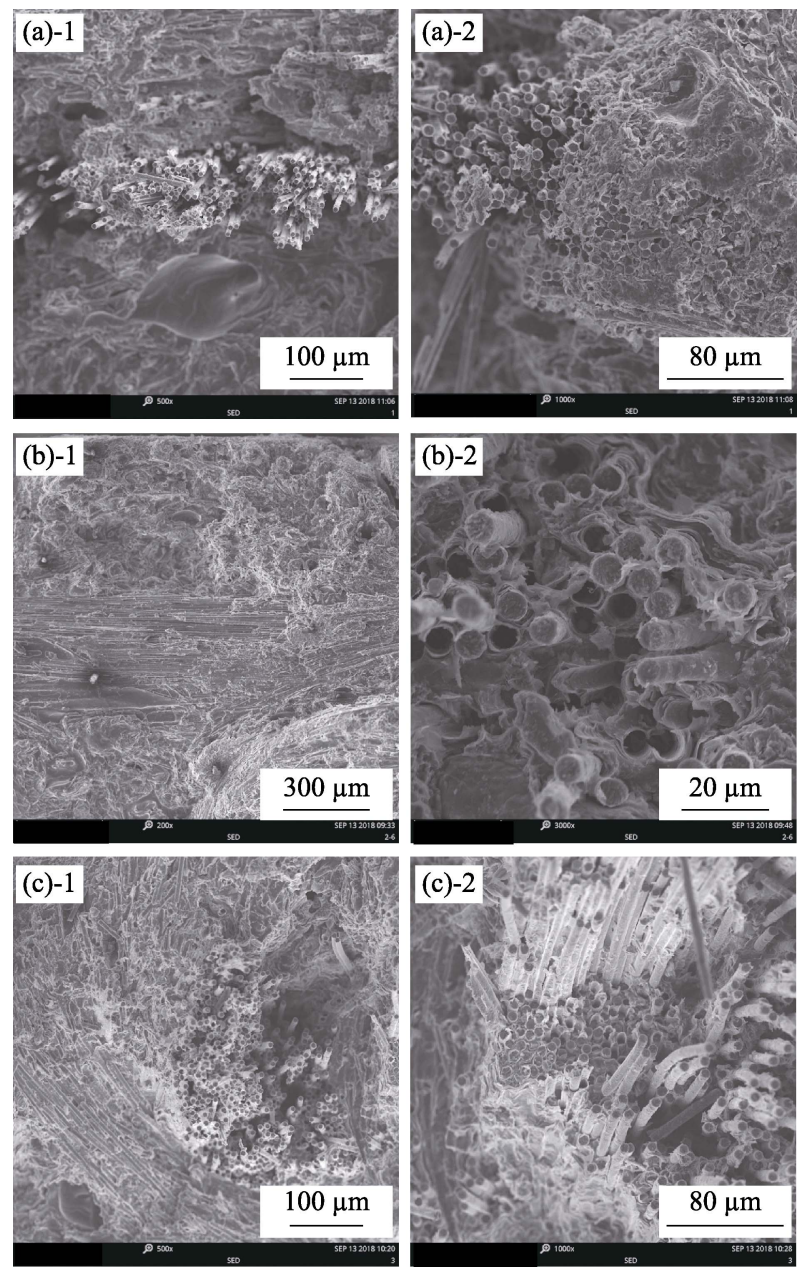

图 5 不同长/短纤维比例碳/碳复合材料的拉伸断口 SEM 照片

Fig. 5 Fracture surface micro-morphologies of needle $\mathrm{C} / \mathrm{C}$ composites after tensile tests

(a) A; (b) B-8; (c) C

\subsection{3 热物理性能}

1)热膨胀系数 $(\mathrm{A}, \mathrm{B}-8, \mathrm{C})$

碳/碳复合材料的热膨胀特性直接影响其在高 温环境应用时的抗热冲击、热疲劳以及热匹配能力, 不仅是考察和评价高温服役综合性能的主要依据, 而且是深入研究材料微缺陷、相变等微结构变化的 重要指标。制备碳/碳复合材料温度高(碳化温度 $\left.700 \sim 1000{ }^{\circ} \mathrm{C}^{[17]}\right)$, 预制体结构复杂, 且纤维与基体 热膨胀不匹配，导致在制备过程中存在热应力。材 料及典型构件应力的大小与分布是热结构设计的重 要依据。

对比分析了短/长纤维比例不同、其它结构参数 相同的针刺预制体对应的碳/碳复合材料的面内热 膨胀系数随温度的变化规律, 如图 6 所示。碳/碳复 合材料热膨胀系数随温度的变化均呈现先降低后升 高的趋势。材料在低于 $600{ }^{\circ} \mathrm{C}$ 的温度下表现为负膨 胀, 最大负膨胀系数出现在 $400{ }^{\circ} \mathrm{C}$ 左右。其中, 预 制体密度最高的 $\mathrm{C}$ 材料在 $400{ }^{\circ} \mathrm{C}$ 的热膨胀系数为 $-0.32 \times 10^{-6} /{ }^{\circ} \mathrm{C}$, 在三种材料中最低; 从 $400{ }^{\circ} \mathrm{C}$ 至 $1200{ }^{\circ} \mathrm{C}$ 范围内, 碳/碳复合材料的热膨胀系数呈单 调增大的趋势，预制体密度最低的 $\mathrm{A}$ 材料在 $1200{ }^{\circ} \mathrm{C}$ 的热膨胀系数为 $0.88 \times 10^{-6} /{ }^{\circ} \mathrm{C}$, 在三种材料 中最高。

均质材料的热膨胀系数与材料的晶体结构和结 合能等有关。碳/碳复合材料的热膨胀系数不仅与碳 基体有关，还与预制体的纤维排布、短/长纤维比例 等织物参数有关。对比分析了不同密度预制体的碳/ 碳复合材料发现, 高密度预制体碳/碳复合材料的连 续长纤维多, 低密度预制体碳/碳复合材料的短切纤 维和碳基体多。在材料升温初始阶段, 碳纤维产生 负膨胀收缩，高纤维体积含量的材料收缩明显，表 现为 $\mathrm{C}$ 材料在 $400{ }^{\circ} \mathrm{C}$ 的最大负膨胀; 随着温度升高 到材料变形拐点后, 碳纤维的热膨胀系数低于沥青 碳基体的热膨胀系数, 低纤维体积含量(高碳基体

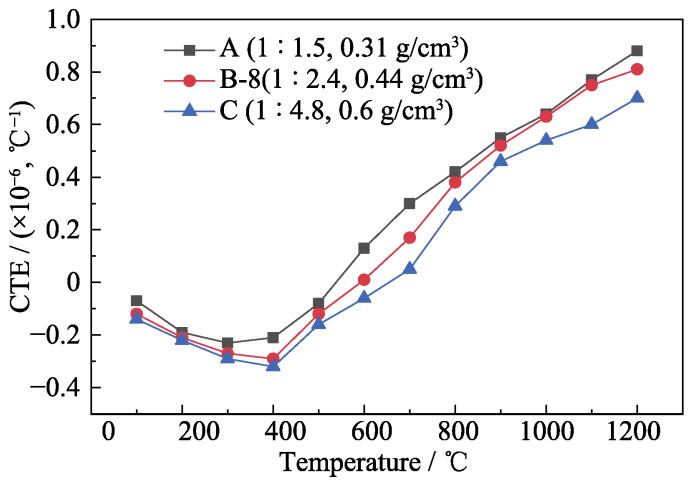

图 6 不同短/长纤维比例碳/碳复合材料的热膨胀系数 Fig. 6 Thermal expansion coefficients of three composites 
含量)的材料膨胀明显, 表现为 $\mathrm{A}$ 材料在 $1200{ }^{\circ} \mathrm{C}$ 的 最大正膨胀。针对高温服役环境, 高纤维体积含量 的材料热膨胀系数相对较低, 表现出更好的高温稳 定性与适用性。

2)导热系数(B-1,B-2,B-3,B-4)

热导率是高温热结构材料的重要技术指标, 在 高温条件下使用时，碳/碳复合材料内部的热场环境 取决于材料的导热性能, 若材料导热系数大, 则材 料内部沿热场方向的热梯度就小, 抗热振性能就 越好。

对比针刺密度不同、其它结构参数相同的预制 体对应的碳/碳复合材料厚度方向的导热性能发现, 材料的导热系数随温度升高而增大, $700{ }^{\circ} \mathrm{C}$ 以上趋 于稳定(图 7)。这是因为碳/碳复合材料为非均质混 合结构，基体介于乱层石墨和晶体石墨之间，导热 机理既有声子导热又有电子导热，在温度较低时, 声子和电子活动加剧, 导热率增大; 但是在较高温 度时, 声子和电子之间的相互作用和碰撞进一步加 强, 偏离平衡位置较大, 平均自由程减小, 形成散 射现象, 使导热系数增幅减小并趋于平稳 ${ }^{[18]}$ 。另外, 从数据规律还能看出, 针刺密度越高的碳/碳复合材 料因厚度方向引入的短切纤维越多, 形成的局域热 短路越多，导热性能越好。

选取导热性能最好的针刺碳/碳复合材料(B-4), 研究材料厚度方向与面内方向热导率差异以及高温 热处理对热导率的影响。结果表明, 其导热特性呈 现各向异性特征, $X 、 Y$ 向(面内)热导率明显大于 $Z$ 向(厚度)热导率, 主要是由于面内方向的连续纤维 含量远远高于厚度方向的连续纤维含量; 另外, 经 $2000{ }^{\circ} \mathrm{C}$ 热处理后(图 8), 材料热导率略微升高, 是 由于碳基体在高温处理后，其石墨化度提高，结构 趋于有序, 致使基体导热性能提高。对高温环境应 用的薄壁热结构而言, 较大的面内热导率有利于热

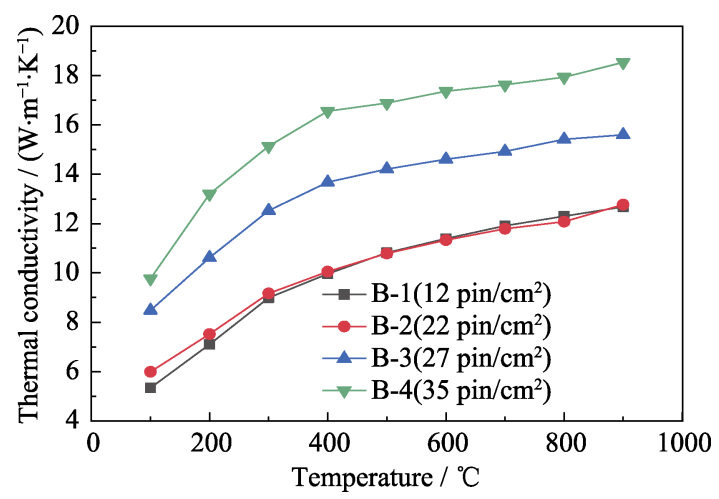

图 7 不同针刺深度碳/碳复合材料的热导率

Fig. 7 Thermal conductivity of four composites with different needling densities

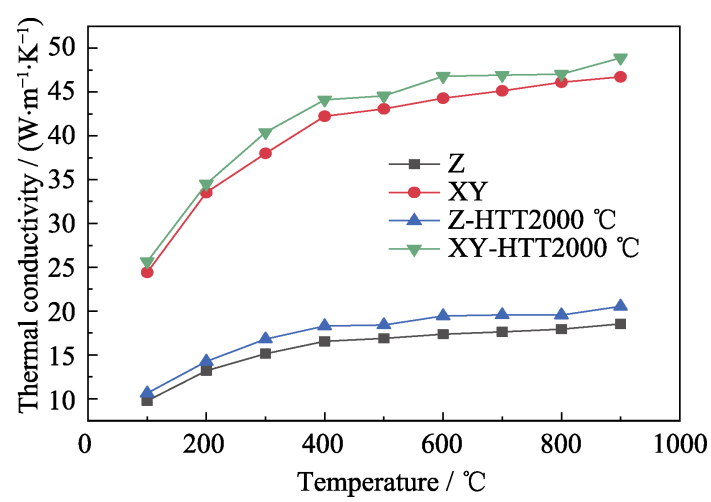

图 8 高温热处理对材料热导率的影响

Fig. 8 Effect of heat treatment on thermal conductivity

量由高温区向低温区传导，避免热结构局部区域温 度过高使材料失效。

\section{3 结论}

1) 以东丽 $\mathrm{T} 700-12 \mathrm{~K}$ 碳纤维为原料, 通过优化 针刺成型参数, 制备了优于传统针刺预制体的高密 度预制体。采用沥青高压浸渍一碳化工艺制备了高性 能针刺碳/碳复合材料, 高密度针刺预制体对应材料 的拉伸、压缩、弯曲、面内剪切和层间剪切强度可 达 207、228、285、54 和 $28 \mathrm{MPa}$;

2) 针刺成型参数对碳/碳复合材料的力学性能 影响显著。针刺深度和针刺密度的增加会降低材料 拉伸、弯曲、压缩和面内剪切性能，并且提高材料 的层间剪切性能; 针刺深度变化对碳/碳复合材料 性能造成的影响效果比针刺密度变化对碳/碳复合 材料性能造成的影响效果更为显著; 提高连续纤维 含量可显著提高材料的面内拉伸和弯曲性能, 对材 料的剪切性能影响不明显;

3) 针刺成型参数对碳/碳复合材料的热物理性 能影响显著。其中, 纤维体积含量越高的碳/碳复合 材料在高温条件下的热膨胀系数越小(越稳定); 针 刺碳/碳复合材料热导率呈各向异性特征，其面内热 导率远高于厚度方向热导率; 针刺密度越高, 材料 厚度方向热导率越高，高温热处理可略微提高材料 的热导率，但效果不明显。

本研究所制备的高纤维体积含量针刺碳/碳复 合材料力学和热物理性能优良, 可向高性能热结构 材料方面推广应用，为高性能热结构材料用预制体 的低成本化提供参考。

\section{参考文献:}

[1] 黄启忠. 高性能针刺炭/炭复合材料的制备、结构与应用. 长沙: 中南大学出版社. 2010: 5-6. 
[2] FITZER E. The future of carbon-carbon composites. Carbon, 1987, 25(2): 163-190.

[3] 李贺军. 炭/炭复合材料. 新型炭材料, 2001, 16(2): 79-80.

[4] SACAGE G. Carbon-carbon composites. London: Chapman and hall, 1993, 323-359.

[5] 左劲旅, 张红波, 熊翔, 等. 喉衬用炭/炭复合材料研究进展. 炭素, 2003 (2): 7-10.

[6] JI A L, LI H J, CUI H, et al. Axial thermodynamic performance analysis of the different preform $\mathrm{C} / \mathrm{C}$ composites. Journal of Inorganic Materials, 2010, 25(9): 994-998.

[7] 孙乐, 王成, 李晓飞, 等. $\mathrm{C} / \mathrm{C}$ 复合材料预制体的研究进展. 航 空材料学报, 2018, 38(2): 86-95.

[8] CHEN X, CHEN L, ZHANG C, et al. Three-dimensional needledpunching for composites - a review. Composites: Part A: Applied Science and Manufacturing, 2016, 85: 12-30.

[9] LACOMBE A. HERAKLES Thermal-thermal-structural Composite Materials Boost Rocket Nozzle Performance. AIAA 2013-3863. 2013.

[10] LACOMBE A, PICHON T, LACOSTE M. High Temperature Composite Nozzle Extensions, a Mature and Efficient Technology to Improve Upper Stage Liquid Rocket Engine Performance. AIAA 2007-5470, 2007.

[11] ELLIS R A, LEE J C, PAYNE F M. Carbon/carbon extendible nozzles. Acta Astronautica, 2002, 50(6): 357-367.

[12] 嵇阿琳, 李贺军, 崔红, 等. 针刺碳纤维预制体的发展与应用. 炭素技术, 2010, 29(3): 23-27.

[13] 张宏波, 姜召阳, 孙陈成, 等. 无纬布增强针刺毡 $\mathrm{C} / \mathrm{C}$ 复合材料 性能的研究. 宇航材料工艺, 2007(1): 19-22.

[14] 孙岩, 刘勇琼, 廖英强, 等. 针刺 $\mathrm{C} / \mathrm{C}$ 复合材料剪切性能. 宇航 材料工艺, 2012(3): 91-95.

[15] 李艳, 崔红, 王斌, 等. 致密化工艺对厚壁针刺 $\mathrm{C} / \mathrm{C}$ 复合材料性 能的影响. 复合材料学报, 2017, 34(10): 2337-2343.

[16] ZHENG J H, LI H J, CUI H, et al. Relations between needling processing parameters and tensile strength of $\mathrm{C} / \mathrm{C}$ composites. Journal of Inorganic Materials, 2017, 32(11): 1147-1153.

[17] 熊翔, 黄伯云, 李江鸿, 等. 准三维 $\mathrm{C} / \mathrm{C}$ 复合材料的弯曲性能及 其破坏机理. 航空材料学报, 2006, 26(4): 88-91.

[18] MANOCHA L M. Thermophysical properties of densified pitch based carbon/carbon materials-- II. Bidirectional composites. Carbon, 2006, 44(3): 480-487. 